\title{
Knockdown of Sucla2 decreases the viability of mouse spermatocytes by inducing apoptosis through injury of the mitochondrial function of cells
}

\author{
Shaoping Huang, ${ }^{1,2}$ Jing Wang ${ }^{3}$, Lei Wang ${ }^{2}$ \\ ${ }^{1}$ Department of Human Anatomy and Neuroscience, Medical School, Southeast University, \\ Nanjing, China \\ ${ }^{2}$ State Key Laboratory of Reproductive Medicine, Nanjing Medical University, Nanjing, China \\ ${ }^{3}$ MingDe Hospital affiliated with Nanjing Medical University, Nanjing, China
}

\begin{abstract}
Introduction. Sucla2, a $\beta$ subunit of succinyl coenzyme A synthase, is located in the mitochondrial matrix. Sucla2 catalyzes the reversible synthesis of succinate and adenosine triphosphate (ATP) in the tricarboxylic acid cycle. Sucla 2 expression was found to be correlated with the capacitation of boar spermatozoa. We have previously reported that Sucla2 was decreased in the testes of rats with spermatogenesis failure after exposure to endocrine disruptor BDE47. Yet, the expression model of Sucla2 in spermatogenesis and the function of Sucla2 in spermatogenic cells are still unclear. Our objective was to explore the localization of Sucla2 during mouse spermatogenesis and its function in the mouse spermatocyte line (GC2).

Material and methods. The localization of Sucla2 in the mouse testis was explored through immunohistochemistry (IHC). Sucla2 was knocked down in GC2 cells and its expression was detected by Western blot (WB) to verify the efficiency of the siRNA transfection. Mitochondrial membrane potential (MMP), apoptosis and ROS of GC2 were detected by flow cytometry. ATP production was measured by the luminometric method and the presence of Bcl2 of GC2 was detected by WB.

Results. Sucla2 is highly expressed in all germ cells but not in interstitial cells. Coarse Sucla2 signals are found in spermatocytes in stages VII-XII of mouse spermatogenesis. In GC2 cells, knockdown of Sucla2 decreased cell viability and MMP, induced apoptosis of GC2 cells, decreased ATP production, and Bcl2 expression, and increased ROS levels.

Conclusions. Sucla2 is related to the developmental stages of mouse spermatogenesis. Knockdown of Sucla2 decreases the viability of mouse spermatocytes by inducing apoptosis via decreased mitochondrial function of the cells. (Folia Histochemica et Cytobiologica 2016, Vol. 54, No. 3, 134-142)
\end{abstract}

Key words: mouse; Sucla2 knockdown; spermatogenesis; spermatocyte; mitochondria; apoptosis; Bcl2; ROS; ATP

\section{Introduction}

Sucla2, a $\beta$ subunit of Succinyl coenzyme A synthase, is located in the mitochondria and catalyzes the reversible synthesis of succinate and adenosine triphosphate (ATP) in the tricarboxylic acid cycle. Sucla2 is encoded

Correspondence address: S. Huang, Ph.D.

Department of Human Anatomy and Neuroscience

Medical School, Southeast University, China

tel.: 86-25-83272505

e-mail: huangsp-8888@163.com by the nuclear gene SUCLA2 [1], and is associated with the maintenance of mitochondrial DNA (mtDNA) and function of mitochondria [2]. Defects in the SUCLA2 gene result in myopathic mtDNA depletion syndrome, characterized by a significant reduction in mtDNA levels and mitochondrial dysfunction [3-5]. In patients with SUCLA2 mutation, impaired production of ATP and high levels of reactive oxygen species (ROS) were found in fibroblasts [6]. Only a few authors have studied Sucla2 expression in males. Recently, proteomic analysis showed that Sucla2 is related to capacitation of boar spermatozoa [7]. 
In a previous study, we found that the protein level of Sucla2 was decreased in testes of rats with spermatogenesis failure, and decreased in mouse spermatogonia cell line (GC1) with increased apoptosis after exposure to the endocrine disruptor BDE47; Sucla2 is located in germ cells and is highly expressed in VII-XII stages of rat spermatogenesis [8]. This previous study indicates that Sucla2 is important during spermatogenesis, and probably plays an important role in the proliferation and apoptosis of germ cells. Nonetheless, the function of Sucla2 in mammalian spermatogenic cells has not yet been investigated and the precise localization of the Sucla2 protein in mouse testis is still unclear. The aim of our study was thus to explore the function and expression of Sucla2 during mouse spermatogenesis through knockdown of its mRNA in the mouse spermatocyte cell line (GC2).

\section{Material and methods}

Animals and treatment. Adult male ICR mice $(28 \pm 2 \mathrm{~g})$ were obtained from the Laboratory Animal Center of Nanjing Medical University (Nanjing, China) and maintained in a 12/12-h light/dark cycle at $20-22^{\circ} \mathrm{C}$ and $50-70 \%$ humidity, with food and water provided ad libitum. The animals were treated according to the rules of the Nanjing Medical University and sacrificed with carbon dioxide. Testes were collected and fixed in Bouin's solution. The study was approved by The Animal Ethical Committee of Nanjing Medical University, Nanjing, China; study number: 20090598). The fixed tissues were embedded in paraffin, sectioned, and stained as described before [9].

Immunohistochemistry (IHC). IHC was used to explore the localization of Sucla 2 in mouse testes. Paraffin sections of mouse testes were immunostained as described previously [9]. In brief, deparaffinated sections of mouse testis were incubated in $2 \% \mathrm{H}_{2} \mathrm{O}_{2}$ to quench the endogenous peroxidase activity, washed in phosphate-buffered saline (PBS), blocked with a blocking serum and incubated with primary antibody against Sucla2 (1:50, 12627-1-AP, ProteinTech Group Inc., Chicago, IL, USA) overnight at $4^{\circ} \mathrm{C}$. The next day, sections were washed with PBS, and incubated with HRP-conjugated secondary antibody (1:1500, ab97080, abcam, Cambridge, MA, USA). The immunoreactive sites were visualized as brown staining with diaminobenzidine and slides were mounted for bright-field microscopy (Axioskop 2 plus, Zeiss, Jena, Germany).

Cell culture. GC2 cells (mouse spermatocyte line, GC2) were obtained from ATCC Global Bioresource Center (ATCC Global Bioresource Center, Atlanta, GA, USA). Cells were cultured in Dulbecco's modified Eagle's medium (DMED) supplemented with $10 \%$ fetal bovine serum, $1 \%$ penicillin/streptomycin, and $25 \mu \mathrm{g} / \mathrm{mL}$ ascorbic acid at $37^{\circ} \mathrm{C}$ in an atmosphere containing $5 \% \mathrm{CO}_{2}$ (all culture reagents were obtained from Sigma, St. Louis, MO, USA) [10]. Cell viability and counts were determined by trypan blue exclusion. GC2 cells were suspended in $0.04 \%$ trypan blue for $3 \mathrm{~min}$, assuming that dead cells were stained in blue while viable cells were not. Cell viability was expressed as a percentage reflecting the number of viable cells relative to the number of viable cells in the control [11].

\section{Small interference RNA (siRNA) transfection of GC2 cells.}

To explore the function of Sucla2 in germ cells, Sucla2-targeting siRNA transfection was used to knock down Sucla2 in GC2 cells. In order to explore the relationship between different Sucla2 levels and their effects on GC2 cells, different volumes of Sucla2-targeting siRNA was used to knock down Sucla2. In brief, control siRNA (sc-37007, Santa Cruz Biotech, Santa Cruz, CA, USA), Sucla2 siRNA (sc-76599; Santa Cruz Biotech) against the mouse Sucla2 mRNA were diluted to a final concentration of $10 \mu \mathrm{M}$ and stored at $-20^{\circ} \mathrm{C}$. The GC2 cells were seeded in six well culture plates and cultured for 24 hours until cells were $60-80 \%$ confluent. Cells were washed once with $2 \mathrm{~mL}$ transfection medium. For the control (Neg), added $2 \mathrm{~mL}$ transfection medium containing $12 \mu \mathrm{L}$ transfection reagent (11668-019, Invitrogen Corporation, Carlsbad, CA, USA) and $12 \mu \mathrm{L}$ control siRNA; in the Si1 group, added $2 \mathrm{~mL}$ transfection medium containing $12 \mu \mathrm{L}$ transfection reagent, $6 \mu \mathrm{L}$ control siRNA and $6 \mu \mathrm{L}$ Sucla2 siRNA; in Si2 group, added $2 \mathrm{~mL}$ transfection medium containing $12 \mu \mathrm{L}$ transfection reagent and $12 \mu \mathrm{L}$ Sucla 2 siRNA. Cells were cultured at $37^{\circ} \mathrm{C}$ for $6 \mathrm{~h}$ in a $\mathrm{CO}_{2}$ incubator. The medium was then discarded and $2 \mathrm{~mL}$ of normal growth medium was added. The cells were incubated for additional $24 \mathrm{~h} \mathrm{[10].} \mathrm{Cells} \mathrm{were} \mathrm{collected} \mathrm{for}$ further analysis.

Protein extraction. After siRNA transfection, the GC2 cells were collected and homogenized in a lysis buffer containing $1 \%(\mathrm{v} / \mathrm{w})$ protease inhibitor cocktail (78415, Pierce Biotechnology, Rockford, IL, USA). The mixture was shaken at $4^{\circ} \mathrm{C}$ for $1 \mathrm{~h}$, and the insoluble matter was removed by centrifugation at $40,000 \mathrm{~g}$ and $4^{\circ} \mathrm{C}$ for 1 hour. Protein concentrations were determined by the Bradford method using BSA as the standard [8].

Western blot analysis. Protein levels of Sucla2 and $\mathrm{Bcl} 2$ in GC2 after siRNA transfection were analyzed by Western blot, as previously described [12]. Glyceraldehyde-3-phosphate dehydrogenase (Gapdh) was used as an internal reference. Antibodies against Sucla2 (1:1500, 12627-1-AP, ProteinTech Group, Inc., Chicago, IL, USA), Bcl-2 (1:50, sc-7382, Santa Cruz Biotech), and Gapdh (1:1000, Kc-5G5, KangChen Bio-tech Inc., Shanghai, China) were purchased. Protein samples were electrophoresed on $12 \%$ SDS poly- 
acrylamide gels and transferred to nitrocellulose membranes (Amersham Biosciences AB, Uppsala, Sweden). Membranes were then blocked in phosphate-buffered saline (PBS) containing 5\% nonfat milk powder for $1 \mathrm{~h}$, and incubated overnight with anti-Sucla2, anti-Bcl2, or anti-GAPDH primary antibody in PBS containing 5\% nonfat milk powder. The next day, membranes were washed three times (10 min each) with PBS and incubated with horseradish peroxidase (HRP)-conjugated secondary antibody (Beijing ZhongShan Biotechnology Co. Ltd., Beijing, China) at $37^{\circ} \mathrm{C}$ for $1 \mathrm{~h}$. Specific proteins were detected using an ECL kit (Amersham Biosciences, Buckinghamshire, England) and AlphaImager (FluorChem5500; Alpha Innotech, San Leandro, CA, USA).

Detection of mitochondrial membrane potential (MMP). MMP was detected by flow cytometry using JC-1 mitochondrial potential detection kit (C2006, Beyotime Biotech, Shanghai, China) as previously indicated [8]. In brief, GC2 cells after Sucla2-targeting SiRNA transfection were incubated with JC- 1 dye for $15 \mathrm{~min}$ in DMEM at $37^{\circ} \mathrm{C}$, and then subjected to flow cytometry. Healthy cells emitted red fluorescence, while cells with disrupted MMP emitted green fluorescence. The red/green fluorescence ratio ( $\mathrm{Y} / \mathrm{X}$ ratio) is an indicator of MMP: a lower ratio indicates mitochondrial depolarization. The data were analyzed with Cell Quest software (Becton Dickinson, Franklin Lakes, NJ, USA).

Determination of apoptotic GC2 cells. Sucla2 siRNA transfection apoptotic GC2 cells were detected by flow cytometry (Becton Dickinson, FACS Canto, Franklin Lakes, NJ, USA) using the Annexin V-FITC/PI Apoptosis Detection Kit (Bll-AP0103, Biouniquer Technology, Hongzhou, China). FITC-labeled Annexin V binds phosphatidylserine on the surface of apoptotic cells, but of not living cells, whereas PI can be excluded by living cells. The Annexin V-positive/ /PI-negative cells (lower right quadrant, LR) were early apoptotic cells, the double-positive (upper right quadrant, UR) were late apoptotic cells, the Annexin V-negative/ /PI-positive (upper left quadrant, UL) were dead/necrotic cells, and the double-negative cells (lower left quadrant, LL) were viable cells. The sum of LR and UR is the percentage of apoptotic GC2 cells [13].

Determination of ATP levels. GC2 cells were collected and washed twice, before being homogenized in lysis buffer containing $1 \%(\mathrm{v} / \mathrm{w})$ protease inhibitor cocktail (78415, Pierce Biotechnology, Rockford, IL, USA). The mixture was shaken at $4^{\circ} \mathrm{C}$ for 1 hour, and then centrifuged at $40,000 \mathrm{~g}$ for 1 hour at $4^{\circ} \mathrm{C}$. The supernatant was subjected to ATP measurement by luminometric methods, using an ATP assay kit (s0026, Beyotime, Shanghai, China) and a luminometer (TD-20/20, Turner Designs, Sunnyvale, CA, USA) as previously described [8]. ATP levels were also normalized to protein level to exclude the effect of the weight of the cell proteins.
Detection of reactive oxygen species (ROS) production. ROS detection kit (s0033, Beyotime Biotech, Shanghai, China) and flow cytometry were used to detect the reactive oxygen species (ROS) in the cells, as described in a previous paper [14]. 2',7'-dichlorodihydrofluorescein diacetate (DCFH-DA, Sigma-Aldrich, Saint Louis, MO, USA) is a fluorescent dye with no fluorescence. When DCFH-DA enters into cytoplasm of cells, it can be oxidized by ROS to form 2',7'-dichlorodihydrofluorescein (DCF) emits fluorescence allowing ROS levels to be determined through measuring the DCF fluorescence intensity. The cells were collected and incubated with 2',7'-dichlorodihydrofluorescein diacetate (DCFH-DA, Sigma-Aldrich) at $37^{\circ} \mathrm{C}$ for $30 \mathrm{~min}$, washed with PBS, and analyzed by flow cytometry.

Statistical analysis. The numerical data were generated from three independent experiments and expressed as means \pm SDs. ANOVA (with the LSD post-hoc statistical test) was used to evaluate the significant differences between different groups (SSPS 20.0, IBM Co., Armonk, NY, USA). There results were considered to be statistically significant at $P<0.05$

\section{Results}

\section{Immunohistochemistry (IHC) of Sucla2 in mouse testes}

The IHC of Sucla2 was performed because the localization of Sucla2 in the mouse testis is still unclear. We found that Sucla2 is located specifically in the cytoplasm of the Sertoli cells (Ser) and all the spermatogenic cells such as leptotene spermatocytes (Lc), pachytene spermatocytes $(\mathrm{Pc})$, round spermatids (rSt), and elongated spermatids (eSt) (Figure 1). Coarse Sucla2 signals can be observed in the cytoplasm of Pc in stages VII-XII, but there are very faint signals in Leydig cells and other cells present in the interstitium of the testes. The expression of Sucla2 protein in mouse testes is related to the developmental stages of spermatogenesis (Figure 1).

\section{Efficiency of Sucla2-targeting siRNA transfection and viability of GC2 cells under Sucla2 knockdown}

The Western blot technique was used to verify the efficiency of Sucla2-targeting siRNA transfection. Sucla2 expression was reduced through Sucla2 knockdown in GC2 cells; also, Sucla2 was decreased by increasing the Sucla2-targeting siRNA reagent from $6 \mu \mathrm{L}$ in the $\mathrm{Si} 1$ group to $12 \mu \mathrm{L}$ in the $\mathrm{Si} 2$ group (Figure $2 \mathrm{~A}$ ).

In the GC2 cells transfected by Sucla2-targeting siRNA, cell viability in the Neg, Si1 group (treated with $6 \mu \mathrm{L}$ Sucla2-targeting siRNA), and $\mathrm{Si} 2$ group (treated with $12 \mu \mathrm{L}$ Sucla2-targeting siRNA) was $100 \% \pm 3.4 \%, 76.1 \% \pm 5.3 \%$ and $59.1 \% \pm 9.9 \%$, 


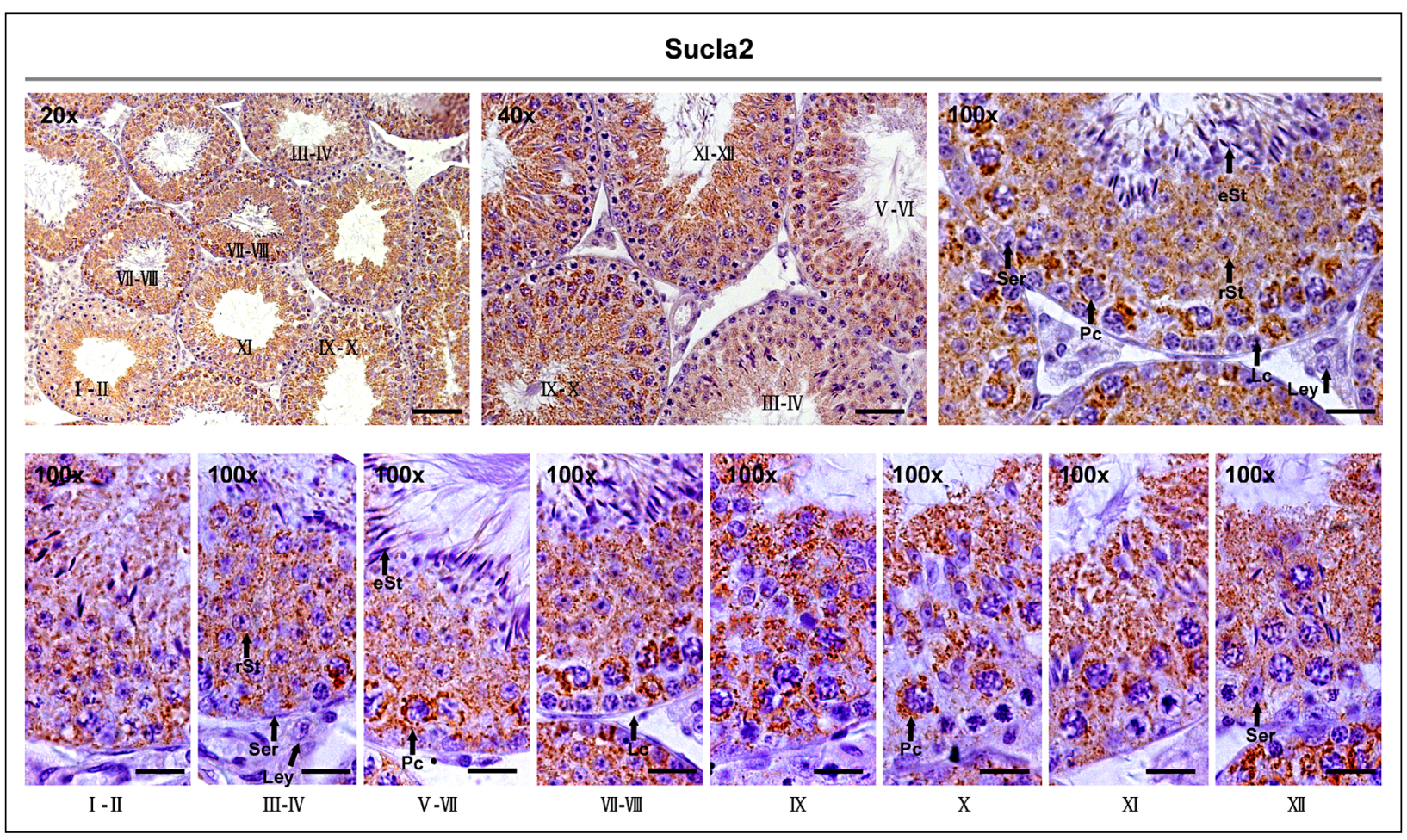

Figure 1. Immunohistochemistry of Sucla2 in mouse testes. Sucla2 is expressed in the cytoplasm of all spermatogenic cells and Sertoli cells, coarse signals can be observed in pachytene spermatocyte in stages VII-XII of spermatogenesis, and very faint signals are observed in Leydig cells. Abbreviations: Lc — leptotene spermatocytes; Pc — pachytene spermatocytes; rSt — round spermatids; eSt — elongated spermatids; Ser — Sertoli cell; Ley — Leydig cell. In $\times 20$, bars $=50 \mu \mathrm{m}$; in $\times 40$, bars $=25 \mu \mathrm{m}$; in $\times 100$, bars $=10 \mu \mathrm{m}$.

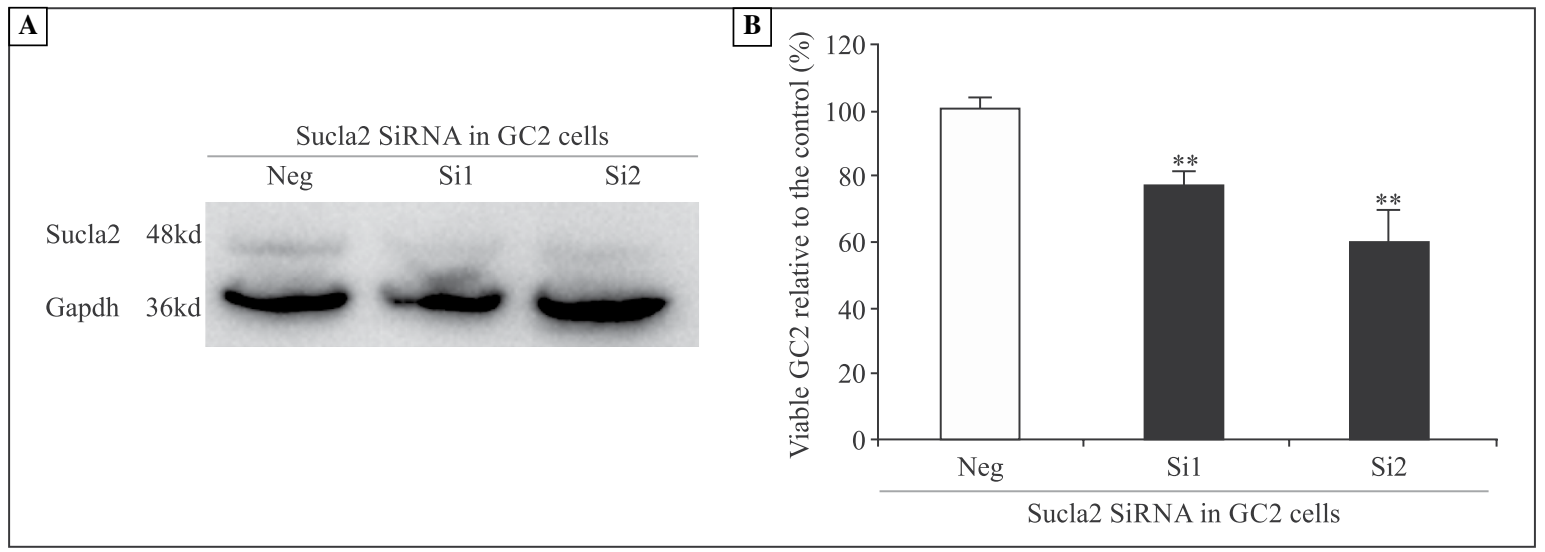

Figure 2. Sucla2 level and viability of GC2 cells under Sucla2 knockdown. GC2 cells were cultured in control (Neg), or $\mathrm{Si}$, or Si2 group transfected with different volumes of Sucla2-targeting siRNA for 6 hours as indicated in Material and methods, then cultured in normal medium for 24 hours. A. Sucla2 levels were determined by Western blotting; B. Viability of GC2 cells under Sucla2 knockdown was determined by trypan blue exclusion. Neg — control cells without siRNA, Si1 and Si2, GC2 cells incubated with 6 or $12 \mu \mathrm{L}$ of Sucla2 siRNA, respectively. Data represent the means \pm SDs from three individual experiments. ${ }^{* *}$ Significantly different from control cells, $p<0.01$.

respectively. Compared with the control, the decrease in cell viability after Sucla2 knockdown was statistically significant in the Si1 and Si2 groups (Figure 2B).

\section{Sucla2 knockdown affects MMP of GC2 cells}

In the Neg (control) group, cells grown in higher density emitted orange fluorescence after JC-1 staining, indicating a higher MMP (orange arrow), 


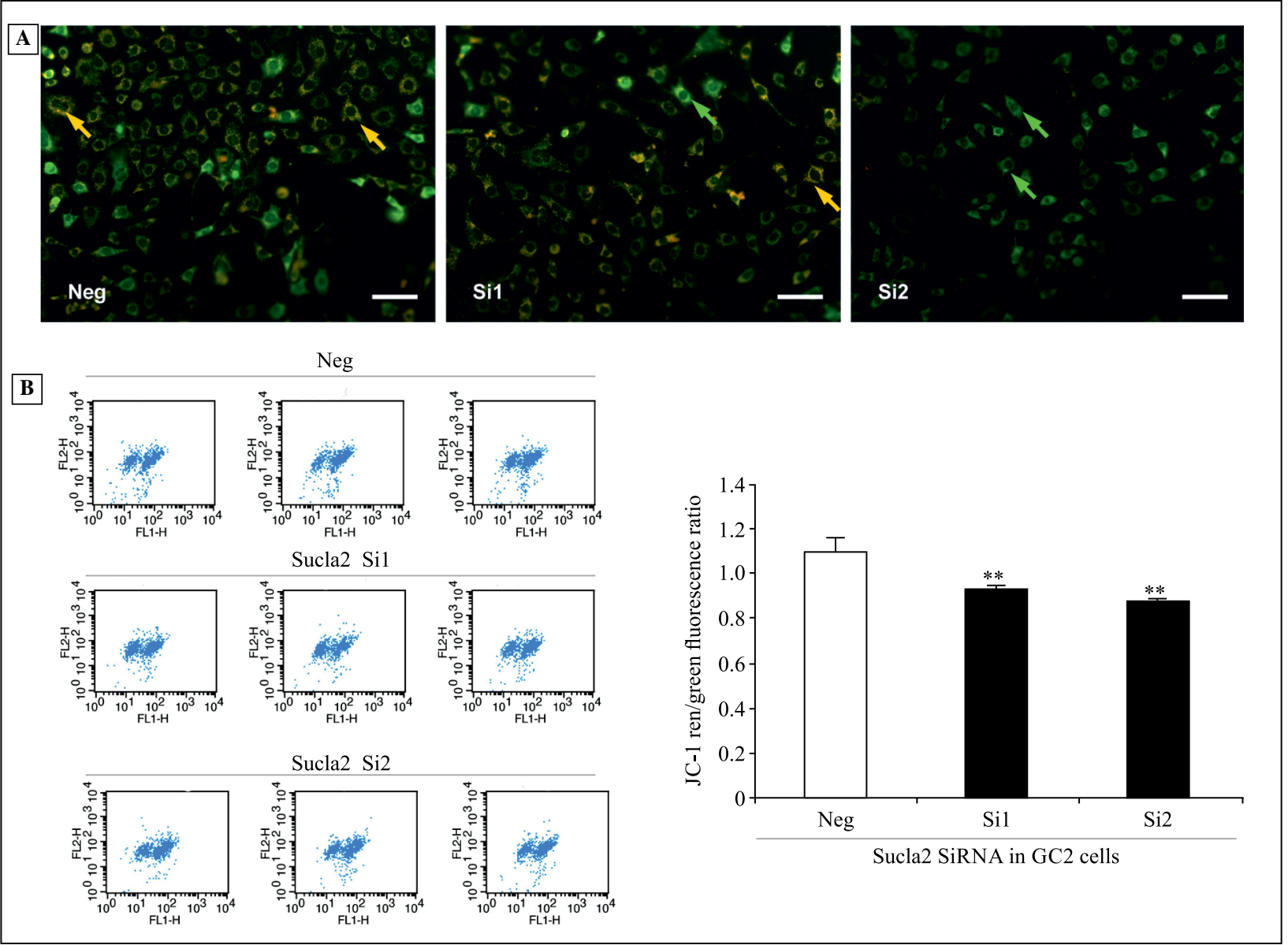

Figure 3. Morphology and mitochondrial membrane potential (MMP) of GC2 cells under Sucla2 knockdown. GC2 cells were cultured as control (Neg) or Si1 or Si2 groups transfected with 6 or $12 \mu \mathrm{L}$ of Sucla2-targeting siRNA, respectively, for 6 hours as indicated in Material and methods, then cultured in normal medium for 24 hours. A. Morphology of GC2 cells under Sucla2 knockdown. Fluorescence microscopy showed that in control cells more cells have high MMP and emit orange fluorescence (orange arrow) whereas in knockdown cells there are more cells with lower MMP that emit green fluorescence (green arrow). Bars $=50 \mu \mathrm{m} ;$ B. MMP of GC2 cells under Sucla2 knockdown was determined by flow cytometry as described in Material and methods. The red/green fluorescence ratio (Y/X ratio) is taken as an indicator of MMP. Data represent the means \pm SDs from three individual experiments. "* Significantly different from control cells, $p<0.01$.

in the Si1 and Si2 groups, cells were smaller in size and grew with lower density, indicating lower MMP (green arrow) and lower viability (Figure 3A). The $\mathrm{red} /$ green fluorescence ratio was $1.09 \pm 0.07,0.92 \pm$ $\pm 0.02,0.87 \pm 0.01$ in the control $(\mathrm{Neg}), \mathrm{Si} 1$ and $\mathrm{Si} 2$ groups, respectively, and the differences $v s$. control were significant (Figure 3B). Thus, the knockdown of Sucla2 decreased MMP in the GC2 cells.

\section{ATP production and ROS level in GC2 cells under Sucla2 knockdown}

The ATP production of GC2 cells in the Neg, Si1, and Si2 groups was $58.39 \pm 3.76,45.48 \pm 2.28,25.25 \pm 6.11$ $\mathrm{nmol} /(\mathrm{L} \cdot \mathrm{mg})$ respectively (Figure $4 \mathrm{~A})$. Compared with the control (Neg) cells, Sucla2 knockdown induced a statistically significant decrease in ATP production in $\mathrm{Si} 1$ and $\mathrm{Si} 2$ cells, respectively.

The average fluorescence intensities of DCF in cells from the Neg, Si1, and Si2 groups were $41.72 \pm$ $\pm 1.13,20.53 \pm 0.47,17.77 \pm 0.3$, respectively. Relative to the Neg (control cells), the increase in ROS production in the Si1 and $\mathrm{Si} 2$ groups was statistically significant (Figure 4B).

\section{Effects of Sucla2 knockdown on apoptosis and Bcl2 level of GC2 cells}

The percentage of apoptotic GC2 cells in the Neg, $\mathrm{Si} 1$, and $\mathrm{Si} 2$ groups was $6.19 \pm 0.68 \%, 6.78 \pm 1.07 \%$, and $9.75 \pm 1.3 \%$, respectively. Compared with the control, the increase of apoptotic cells in the Si2 


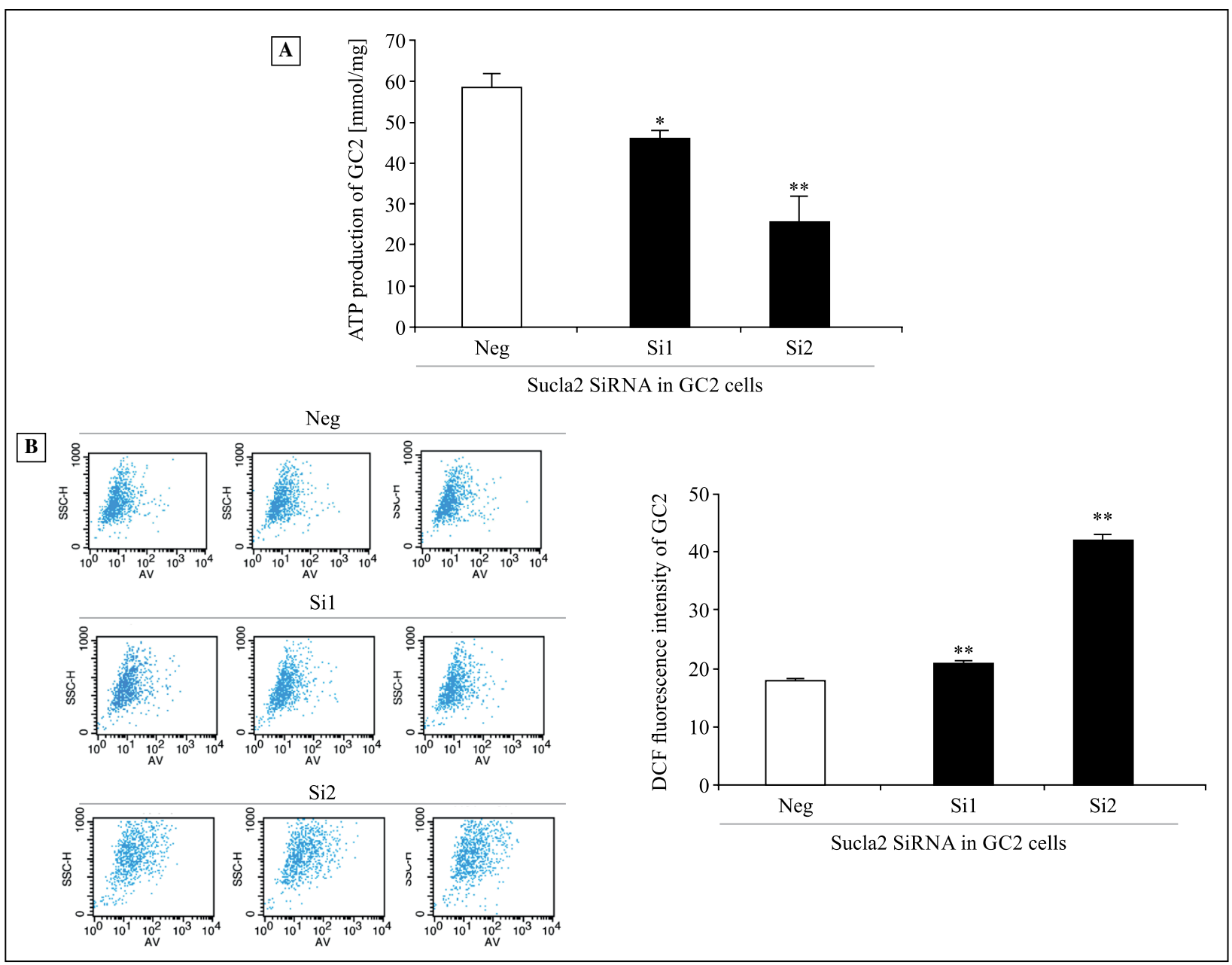

Figure 4. ATP and reactive oxygen species (ROS) levels of GC2 cells under Sucla2 knockdown. GC2 cells were cultured in control (Neg), or Si1 group, or Si2 group transfected with different volumes of Sucla2-targeting siRNA for 6 hours as indicated in Methods, then cultured in normal medium for 24 hours. A. ATP production of GC2 cells under Sucla2 knockdown; B. ROS level of GC2 cells under Sucla2 knockdown. Data represent the means \pm SDs from three individual experiments. ${ }^{* * *}$ Significantly different from control cells, $p<0.05$ and $p<0.01$, respectively.

group was significant, and the data revealed that the knockdown of Sucla2 induces apoptosis in GC2 cells (Figure 5A).

The level of anti-apoptotic factor $\mathrm{Bcl} 2$, as determined by Western blotting, was significantly decreased by Sucla2 knockdown of GC2 cells ( $p<0.01$, Figure 5B). Thus, Sucla2 knockdown induced apoptosis of GC2 cells through the mitochondrial apoptotic pathway.

\section{Discussion}

Spermatogenesis involves the self-renewal of spermatogonial stem cells, differentiation, meiosis of spermatocytes and spermiogenesis [15]. This process is characterized by a delicate balance between cell proliferation and germ cells apoptosis [16]. Mitochon- dria are complex organelles that not only participate in ATP synthesis and ROS production, but also play important roles in proliferation, differentiation, and apoptosis of germ cells $[17,18]$. Mitochondrial function depends not only on mitochondrial molecules encoded by nuclear DNA (nDNA), but also depends on molecules encoded by mitochondria DNA (mtDNA) $[19,20]$. The literature shows that many kinds of abnormal expression of mitochondrial proteins are associated with mitochondrial dysfunction, resulting in mitochondrial diseases such as neurodegeneration, cancer, infertility, and ageing [21-23].

Recently, it has been shown that the abnormal forms of some proteins encoded by nDNA are related to the maintenance of mtDNA. For example, nuclear genetic mutations in EFTu (Tu translation elongation factor, mitochondrial) may result in a disorder of the 


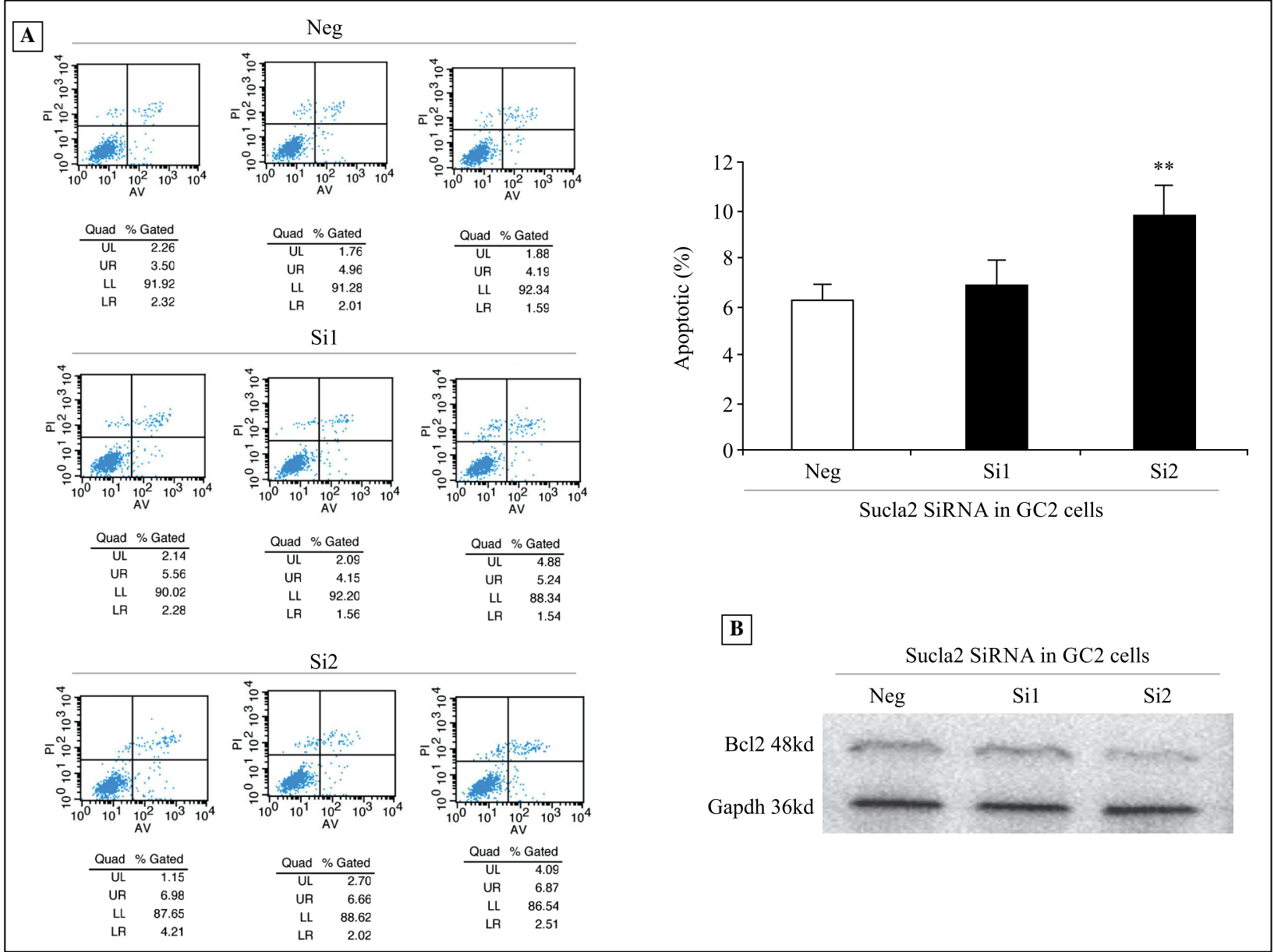

Figure 5. Apoptosis and Bcl2 level of GC2 cells under Sucla2 knockdown. GC2 cells were cultured in control (Neg), or Si1 group, or Si2 group with knockdown Sucla2 for 6 hours as indicated in Material and methods, then cultured in normal medium for 24 hours. A. Apoptosis of GC2 cells under Sucla2 knockdown was studied by flow cytometry. The Annexin V-positive/PI-negative (lower right quadrant, LR) were early apoptotic cells, the double-positive (upper right quadrant, UR) were late apoptotic cells, the Annexin V-negative/PI-positive (upper left quadrant, UL) were dead/necrotic cells, and the double-negative cells (lower left quadrant, LL) were viable cells. The sum of LR and UR is the percentage of apoptotic GC2 cells; B. Bcl2 level of GC2 cells with Sucla2 knockdown was determined by Western blotting. Data represent the means \pm SDs from three individual experiments. ${ }^{* *}$ Significantly different from control cells, $p<0.01$.

mitochondrial respiratory chain [24]; nuclear genetic mutation in $D G U O K$ (mitochondrial deoxyguanosine kinase) [25] or $P O L G$ (mitochondrial DNA polymerase gamma) [24] is associated with mtDNA depletion and disorder of the mitochondria.

Sucla2, encoded by a nuclear gene, is related to mtDNA depletion and mitochondrial dysfunction $[3,26]$. Sucla 2 is the $\beta$ subunit of Succinyl coenzyme A synthase present in the mitochondrial matrix, and catalyzes the reversible synthesis of ATP in tricarboxylic acid cycle. The defects in the SUCLA2 gene can result in myopathic mitochondrial DNA depletion syndrome, which is characterized by a significant reduction in mtDNA content [1]. In a culture medium of fibroblasts from a patient with SUCLA2 mutation,
Western blotting showed a significant decrease in Sucla2 protein and mitochondrial ATP contents and enhanced levels of ROS [6].

Owing to the importance of Sucla2 in mitochondrial function, we focused on its function in the male reproductive system. Our previous study showed that Sucla2 expression decreased in rat testes with failure of spermatogenesis, and decreased in mouse spermatogonia cell line ( $\mathrm{GC} 1$ cells) with an increase in apoptotic cells; moreover, Sucla2 was strongly expressed in spermatocytes of stages VII-XII in rat spermatogenesis [8]. Yet, whether the expression of Sucla 2 in mouse testes is similar to that in rat testes is unknown; also, the function of Sucla2 in the spermatocyte is still unclear. As shown in present study, 
in mouse testis, Sucla2 is located specifically in the cytoplasm of all spermatogenic cells, with coarse signals in the pachytene spermatocytes $(\mathrm{Pc})$ in stages VII-XII and very faint signal in interstitial cells, such as Leydig cells (Lc) (Figure 1). The expression of Sucla2 in mouse testis is similar to that in rat testis; also, Sucla2 is associated with the developmental stages of spermatogenesis. Based on these results, we suspect that Sucla2 is one of the important markers that is decreased by some stresses, and related to mitochondrial dysfunction and the development of germ cells. In present study, Sucla2 was knocked down by Sucla2-targeting siRNA transfection in GC2 cells resulting in decreased cell viability. This finding agrees with the results of our previous study, in which Sucla2 expression was decreased in impaired rat spermatogenesis with an increased ratio of apoptotic germ cells [8].

Mitochondrial membrane potential, a sensitive indicator of mitochondrial function, was determined by flow cytometry. We found that MMP in GC2 cells was reduced significantly by Sucla 2 knockdown; moreover, other indicators of mitochondrial status showed that Sucla2 knockdown decreased ATP and increased ROS contents. An increasing ratio of apoptotic GC2 cells was induced gradually by increasing the Sucla2-targeting siRNA reagent, and $\mathrm{Bcl} 2$, a marker of the mitochondrial apoptotic pathway, was also reduced in cells after Sucla2-targeting siRNA transfection. These results suggest that the knockdown of Sucla2 decreased the viability of spermatocytes by inducing mitochondrial dysfunction and apoptosis of germ cells. Although Sucla2 has been referred to in studies of patients with mitochondrial depletion syndrome (MDS) including progressive external ophthalmoplegia (PEO), ataxia-neuropathy, and mitochondrial neurogastrointestinal encephalomyopathy [26, 27], yet its function in germ cells has rarely been investigated. The present results shed a light on the relationship between Sucla2 and apoptosis or degeneration of male germ cells and encourage further research in this area of study.

Mitochondria are multitasking organelles [28], and many environmental and stress stimuli can cause mitochondrial dysfunction [29, 30], thereby affecting spermatogenesis [31-34]. The present study is a follow-up to our previous works, which showed that Sucla2 expression decreased in testes and GC1 cells with increasing apoptotic cells after exposure to endocrine disruptor BDE47 [8]. Our present data indicate that Sucla2 most likely serves as a link between mitochondrial function and apoptosis of germ cells, and may be one of the targeting markers affected by some stress stimulators. Most studies on Sucla2 focused on the mutation in the SUCLA2 nuclear gene and its relationship with the clinical syndromes of patients $[1,3$, $6,26]$. On the other hand, the majority of studies on the apoptosis of germ cells were focused on the detection of markers of mitochondrial dysfunction or apoptosis, such as ATP production, ROS level, Bcl2, or caspase $3 / 9[8,18,35,36]$. Our studies provide a new way of understanding the mechanism of mitochondrial dysfunction and the apoptosis of germ cells affected by mitochondrial proteins. Based on our findings, Sucla2 is specifically expressed in germ cells and is related to developmental stages of mouse spermatogenesis; knockdown of Sucla2 decreases the viability of mouse spermatocytes, probably by inducing apoptosis through injuring the mitochondrial function of cells.

\section{Acknowledgements}

This study was supported by the Chinese 973 program (2009CB941703) and the Basic Scientific Research Fund, Southeast University of China (2242015K42118; 3224006414).

\section{Conflict of interest}

The authors declare that they have no conflicts of interest.

\section{References}

1. Chinnery PF. Mutations in SUCLA2: A tandem ride back to the Krebs cycle. Brain. 2007;130:606-609. doi: 10.1093/brain/awm023.

2. El-Hattab AW, Scaglia F. Mitochondrial DNA depletion syndromes: Review and updates of genetic basis, manifestations, and therapeutic options. Neurotherapeutics. 2013;10:186-198. doi: 10.1007/s13311-013-0177-6.

3. Venegas V, Halberg MC. Measurement of mitochondrial DNA copy number. Methods Mol Biol. 2012;837:327-335. doi: 10.1007/978-1-61779-504-6-22.

4. Cohen BH, Naviaux RK. The clinical diagnosis of POLG disease and other mitochondrial DNA depletion disorders. Methods. 2010;51:364-373. doi: 10.1016/j.ymeth.2010.05.008.

5. Lamperti C, Fang M, Invernizzi F et al. A novel homozygous mutation in SUCLA2 gene identified by exome sequencing. Mol Genet Metab. 2012;107:403-408. doi: 10.1016/j. ymgme.2012.08.020.

6. Nogueira C, Meschini MC, Nesti C et al. A novel SUCLA2 mutation in a Portuguese child associated with "mild" methylmalonic aciduria. J Child Neurol. 2015;30:228-232. doi: 10.1177/0883073814527158.

7. Kwon WS, Rahman MS, Lee JS et al. A comprehension proteomic approach to identifying capacitation related proteins in boar spermatozoa. BMC Genomics. 2014;15:897. doi: 10.1186/1471-2164-15-897.

8. Huang S, Cui Y, Guo X et al. 2,2',4,4'-Tetrabromodiphenyl ether disrupts spermatogenesis, impairs mitochondrial function and induces apoptosis of early leptotene spermatocytes in rats. Reprod Toxicol. 2015; 51:114-124. doi: 10.1016/j. reprotox.2015.01.009.

9. Shao B, Guo Y, Wang L et al. Unraveling the proteomic profile of mice testis during the initiation of meiosis.J Proteomics. 2015;120:35-43. doi: 10.1016/j.jprot.2015.02.015. 
10. Huang S, Wang J, Cui Y. 2,2',4,4'-Tetrabromodiphenyl ether injures cell viability and mitochondrial function of mouse spermatocytes by decreasing mitochondrial proteins Atp5b and Uqcre1. Environ Toxicol Pharmacol. 2016;46:301-310. doi: 10.1016/j.etap.2016.08.011.

11. Tyagi A, Bhatia N, Condon MS, Bosland MC, Agarwal C, Agarwal R. Antiproliferative and apoptotic effects of silibinin in rat prostate cancer cells. Prostate. 2002;53:211-217. doi: 10.1002/pros.10146.

12. Wang J, Qi L, Huang S et al. Quantitative phosphoproteomics analysis reveals a key role of insulin growth factor 1 receptor (IGF1R) tyrosine kinase in human sperm capacitation. Mol Cell Proteomics. 2015;14:1104-1112. doi: 10.1074/mcp. M114.045468.

13. Razavi R, Gehrke I, Gandhirajan RK, Poll-Wolbeck SJ, Hallek M, Kreuzer KA. Nitric oxide-donating acetylsalicylic acid induces apoptosis in chronic lymphocytic leukemia cells and shows strong antitumor efficacy in vivo. Clin Cancer Res. 2011;17:286-293. doi: 10.1158/1078-0432.CCR-10-1030.

14. Xu T, Pang Q, Zhou D et al. Proteomic investigation into betulinic acid-induced apoptosis of human cervical cancer HeLa cells. PLoS One. 2014;9:e105768. doi: 10.1371/journal. pone. 0105768 .

15. Chen S, Liu Y. Regulation of spermatogonial stem cell self-renewal and spermatocyte meiosis by Sertoli cell signaling. Reproduction. 2015;149:159-167. doi: 10.1530/REP-14-0481.

16. Niu Z, Goodyear SM, Rao S et al. MicroRNA-21 regulates the self-renewal of mouse spermatogonial stem cells. Proc Natl Acad Sci USA. 2011;108:12740-12745. doi: 10.1073/ pnas.1109987108.

17. Yang Y, Karakhanova S, Hartwig W et al. Mitochondria and mitochondrial ROS in cancer: novel targets for anticancer therapy. J Cell Physiol. 2016;231:2570-2581. doi: 10.1002/ jcp. 25349 .

18. Ramalho-Santos J, Varum S, Amaral S, Sousa AP, Amaral A. Mitochondrial functionality in reproduction: from gonads and gametes to embryos and embryonic stem cells. Hum Reprod Update. 2009;15:553-572. doi: 10.1093/humupd/dmp016.

19. Grünewald A, Rygiel KA, Hepplewhite PD, Morris CM, Picard M, Turnbull DM. mitochondrial DNA depletion in respiratory chain-deficient Parkinson disease neurons. Ann Neurol. 2016;79:366-378. doi: 10.1002/ana.24571.

20. Lasserre JP, Dautant A, Aiyar RS et al. Yeast as system for modeling mitochondrial disease mechanisms and discovering therapies. Dis Model Mech. 2015;8:509-526. doi: 10.1242/ dmm.020438.

21. Amaral A, Lourenço B, Marques M, Ramalho-Santos J. Mitochondria functionality and sperm quality. Reproduction. 2013;146:R163-R174. doi: 10.1530/REP-13-0178.

22. Fox TD. Mitochondrial protein synthesis, import, and assembly. Genetics. 2012;192:1203-1234. doi: 10.1534/genetics.112.141267.

23. Herrmann JM, Woellhaf MW, Bonnefoy N. Control of protein synthesis in yeast mitochondria: the concept of translational activators. Biochim. Biophys. Acta. 2013;1833:286-294. doi: 10.1016/j.bbamcr.2012.03.007.

24. Valente L, Tiranti V, Marsano RM et al. Infantile encephalopathy and defective mitochondrial DNA translation in patients with mutations of mitochondrial elongation factors EFG1 and EFTu. Am J Hum Genet. 2007;80:44-58. doi: $10.1086 / 510559$.

25. Ronchi D, Garone C, Bordoni A et al. Next-generation sequencing reveals DGUOK mutations in adult patients with mitochondrial DNA multiple deletions. Brain. 2012;135:3404-3415. doi: 10.1093/brain/aws258.

26. Donti TR, Stromberger C, Ge M, Eldin KW, Craigen WJ, Graham BH. Screen for abnormal mitochondrial phenotypes in mouse embryonic stem cells identifies a model for succinylCoA ligase deficiency and mtDNA depletion. Dis Model Mech. 2014;7:271-280. doi: 10.1242/dmm.013466.

27. Copeland WC. Inherited mitochondrial diseases of DNA replication. Annu Rev Med. 2008;59:131-146. doi: 10.1146/ annurev.med.59.053006.104646.

28. Margineantu DH, Hockenbery DM. Mitochondrial functions in stem cells. Curr Opin Genet Dev. 2016;38:110-117. doi: 10.1016/j.gde.2016.05.004.

29. Shaughnessy DT, McAllister K, Worth L et al. Mitochondria, energetics, epigenetics, and cellular responses to stress. Environ Health Perspect. 2014;122:1271-1278. doi: 10.1289/ /ehp.1408418.

30. Delbès G, Hales BF, Robaire B. Toxicants and human sperm chromatin integrity. Mol Hum Reprod. 2010;16:14-22. doi: 10.1093/molehr/gap087.

31. Lucas BE, Fields C, Joshi N, Hofmann MC. Mono-(-2-ethylhexyl)-phthalate (MEHP) affects ERK-dependent GDNF signalling in mouse stem-progenitor spermatogonia. Toxicology. 2012;299:10-19. doi: 10.1016/j.tox.2012.04.011.

32. Liu C, Duan W, Li R et al. Exposure to bisphenol A disrupts meiotic progression during spermatogenesis in adult rats through estrogen-like activity. Cell Death Dis. 2013;4:e676. doi: 10.1038/cddis.2013.203.

33. Kolasa-Wolosiuk A, Misiakiewicz-Has K, Baranowska-Bosiacka I, Gutowska I, Wiszniewska B. Androgen levels and apoptosis in the testis during postnatal development of finasteride-treated male rat offspring. Folia Histochem Cytobiol. 2015;53:236-248. doi: 10.5603/fhc.a2015.0025.

34. Rajender S, Rahul P, Mahdi AA. Mitochondria, spermatogenesis and male infertility. Mitochondrion. 2010;10:419-428. doi: 10.1016/j.mito.2010.05.015.

35. Hagenaars A, Vergauwen L, Benoot D, Laukens K, Knapen D. Mechanistic toxicity study of perfluorooctanoic acid in zebrafish suggests mitochondrial dysfunction to play a key role in PFOA toxicity. Chemosphere. 2013;91:844-856. doi: 10.1016/j. chemosphere.2013.01.056.

36. Mashayekhi V, Tehrani KH, Hashemzaei M, Tabrizian K, Shahraki J, Hosseini MJ. Mechanistic approach for the toxic effects of perfluorooctanoic acid on isolated rat liver and brain mitochondria. Hum Exp Toxicol. 2015;34:985-996. doi: 10.1177/0960327114565492.

Submitted: 31 May, 2016

Accepted after reviews: 26 September, 2016 Available as AoP: 3 October, 2016 